\title{
Type-II seesaw mass models and baryon asymmetry
}

\author{
Amal Kr. Sarma ${ }^{a, b}$, H. Zeen Devi ${ }^{a}$ and N. Nimai Singh ${ }^{a *}$ \\ ${ }^{a}$ Department of Physics, Gauhati University, Guwahati-781014, India. \\ ${ }^{b}$ Department of Physics, D. R. College, Golaghat-785621, India.
}

\begin{abstract}
We compute and also compare the contributions of canonical and noncanonical mass terms towards baryon asymmetry by considering type-II seesaw mass models of neutrinos: Degenerate(3 varieties), Normal hierarchical and Inverted hierarchical(2 varieties). We have shown that for particular choices of parameter ' $\gamma$ ' ( the so-called discriminator) for different neutrino mass models, the baryon asymmetry is largely dominated by canonical term. Within such type-II seesaw scenario, we find normal hierarchical (NHT3) mass model as the most favourable choice of nature.

Keywords: seesaw neutrino mass, Yukawa coupling, lepton and baryon asymmetry.

PACS numbers: 14.60. Pq, 11.30. Er, 11.30.Fs, 13.35. Hb.
\end{abstract}

\section{Introduction}

The search for a suitable mass model consistent with the observations: masses, mixings and baryon asymmetry, is a standing agenda of present day neutrino physics. With this motivation different neutrino mass models[1,2] were constructed on the basis of celebrated seesaw mechanism[3]. All the models were checked for masses, mixings and stability under radiative corrections. In the electroweak baryogenesis scenario[4,5], the heavy right-handed Majorana neutrinos play a decesive role. In this model of baryogenesis, lepton asymmetry is produced by the decay of lightest of heavy Majorana neutrinos, and nonperturbative sphaleron[6] processes convert this lepton asymmetry to baryon asymmetry. Again the heavy Majorana neutrinos are coupled to light left handed neutrinos via seesaw mechanism. In that sense any mass model constructed on the basis of seesaw mechanism should have the capacity to explain the observed baryon asymmetry. Sakharov's three conditions[7] for baryogenesis: (1) baryon number violation, (2) $\mathrm{C}$ and $\mathrm{CP}$ violation and (3) out of

*Regular Associate of the Abdus Salam ICTP, Trieste, Italy 
equilibrium decay can be realised by the decay of heavy right handed Majorana neutrinos. Recently a non-thermal scenario of baryogenesis for these models was considered[8], where our estimation[9] of baryon asymmetry for different mass models within the thermal leptogenesis scenario was extensively considered. One point missed in ref.(9) is that all the calculations to estimate the baryon asymmetry were confined to type-I seesaw term only, the contributions of non-canonical term were not considered. For completeness we compute the contribution of type-II term also.

We address in this paper, the estimation of baryon asymmetry of the universe using the parameters which are already fixed at the seesaw stage within a common framework. This may possibly discriminate the correct pattern of neutrino mass model in question.

In section 2 we review the discrimination of neutrino mass models in type-II seesaw framework. In section 3, with a brief discussion on the expressions for lepton and baryon asymmetry, we present our numerical calculations and results. Section 4 concludes with a summary and discussion. The zeroth-order mass matrices for various models are collected in Appendix-A. Important expressions related to $m_{L L}^{I}$ and $M_{R R}$ for three neutrino mass models are relegated to Appendix-B.

\section{Type-II seesaw mechanism and discrimina- tion of neutrino mass models}

The type-I seesaw formula relates the light left-handed Majorana neutrino mass matrix $m_{L L}^{I}$ and heavy right handed Majorana mass matrix $M_{R R}$ in a simple way:

$$
m_{L L}^{I}=-m_{L R} M_{R R}^{-1} m_{L R}^{T}
$$

Here, $m_{L R}$ is the Dirac neutrino mass matrix. In some L-R symmetric theories such as $\mathrm{SO}(10)$ GUT, the left-handed Higgs triplet $\Delta_{L}$ acquires vacuum expectation value. Taking this contribution, seesaw formula can be modified as[10]: $m_{L L}=m_{L L}^{I}+m_{L L}^{I I}$ where, the first term is the usual canonical seesaw term (1) and the second term can be expressed as $m_{L L}^{I I}=\gamma\left(M_{W} / v_{R}\right)^{2} M_{R R}$. Such non-canonical seesaw formula (generally known as type-II seesaw formula) can be written as:

$$
m_{L L}=-m_{L R} M_{R R}^{-1} m_{L R}^{T}+\gamma\left(M_{W} / v_{R}\right)^{2} M_{R R} .
$$

In the light of above type-II seesaw formula, the neutrino mass matrices $m_{L L}$ are realised for three different situations: (1) $m_{L L}^{I I}>>m_{L L}^{I}$, (2) $m_{L L}^{I I} \simeq m_{L L}^{I}$ and (3) $m_{L L}^{I I}<<m_{L L}^{I}$. It has been reported that the inequality (1) can naturally provide a connection [11] between the large atmospheric mixing and $b-\tau$ unification in the context of the minimal supersymmetric $\mathrm{SO}(10)$ theory[12]. But we are interested to study the relative strength of these two contributions in all three cases. The second term denoted by $m_{L L}^{I I}$ is heavily constrained by the definition of $v_{R}$, and $v_{R}$ can be extracted from $M_{R R}$ appeared in $m_{L L}^{I}$, thus indicating the dependence of $m_{L L}^{I I}$ on $m_{L L}^{I}$. This point is addressed in the present work. 
The solar and atmospheric neutrino oscillation experiments usually measure only the mass-square differences and in general we have three possible patterns of neutrino masses[13]: (a) Degenerate $m_{1} \simeq m_{2} \simeq m_{3}$, (b) Inverted hierarchical $m_{1} \simeq m_{2}>>m_{3}$ and (c) Normal hierarchical $m_{1}<<m_{2}<<m_{3}$. Depending upon the relative CP-phase of $\left(m_{1}, m_{2}\right)$ pair, the degenerate and inverted hierarchical models are further classified as: degenerate type-1A (DegT1A), degenerate type-1B (DegT1B), degenerate type-1C (DegT1C), inverted hierarchical Type-2A (InvT2A) and inverted hierarchical Type-2B (InvT2B), and these zeroth-order left-handed Majorana mass matrices[2,13] are collected in Appendix A for ready reference. The present forms $[1,2]$ of various mass models given in Appendix B, are derived from seesaw mechanism(1).

The vacuum expectation values $v_{L}$ and $v_{R}$ of left and right-handed Higgs triplets are connected[14] to $\mathrm{W}$-boson mass via $v_{L} v_{R}=\gamma M_{W}^{2}$ where the free parameter $\gamma$ depends on various couplings. Without fine tuning, we generally have $\gamma \sim 1$, but we are searching the input values of $\gamma$ where the contribution of second term arising from the presence of left-handed Higgs triplets is just sufficient to restore the good predictions of $m_{L L}^{I}$ already acquired[2] in type-I seesaw framework. If the second term is dominant, the relevant good predictions of $m_{L L}^{I}$ are spoiled. This can be avoided with the proper choice of $\gamma$ values. We call this $\gamma$ parameter as 'discriminator' of the models. A neutrino mass model is said to be favourable and hence stable when its canonical term dominates over the non-canonical(perturbative) term, and this condition is used here as a criterion for discriminating neutrino mass models[2].

The Dirac neutrino mass matrix $m_{L R}$ appeared in seesaw formula can have any arbitrary structure which either is diagonal or non-diagonal, and it plays a significant role[1] in the construction of $m_{L L}^{I}$. In the seesaw mechanism, for a specific structure of $m_{L L}^{I}$, we can have three possible combinations of $m_{L R}$ and $M_{R R}$ : (a) both $m_{L R}$ and $M_{R R}$ are non-diagonal, (b) $m_{L R}$ diagonal and $M_{R R}$ non-diagonal, (c) $m_{L R}$ non-diagonal and $M_{R R}$ diagonal. These three combinations can be realised in different physical situations. For example, when one calculate lepton asymmetry, one needs to consider the diagonal basis of heavy right-handed neutrinos, and combination (c) becomes relevant. In the present calculations, the diagonal form of $m_{L R}$ is chosen for different neutrino mass matrices. To see this let us consider[2] the seesaw relation $m_{L L}^{I}=-m_{L R} M_{R}^{-1} m_{L R}^{T}$, where both $m_{L R}$ and $M_{R}$ are non-diagonal. Using some left and right handed rotations, the Dirac neutrino mass matrix can be diagonalised[15] as $m_{L R}^{\text {diag }}=U_{L} m_{L R} U_{R}^{\dagger}$. In terms of diagonal basis of $m_{L R}$, the seesaw relation reduces to

$$
m_{L L}^{I}=-U_{L}^{\dagger} m_{L R}^{\operatorname{diag}} M_{R R}^{-1} m_{L R}^{\operatorname{diag}} U_{L}^{*},
$$

where, $M_{R R}^{-1}=U_{R} M_{R}^{-1} U_{R}^{T}$. It is assumed that eigenvalues of $m_{L R}^{\text {diag }}$ are hierarchical (similar to quarks or charged leptons). In absence of Dirac left handed rotations[15], we can set $U_{L} \sim 1$. For slight deviation from unity we can assume $U_{L} \simeq U_{C K M}$, where $U_{C K M}$ is the quark mixing matrix. Again this can be set 
to unity as quarks mixings are very small. This type of approximations do not produce significant change in numerical calculations. For $U_{L} \sim 1$ the eq.(3) reduces to $m_{L L}^{I}=-m_{L R}^{\text {diag }} M_{R R}^{-1} m_{L R}^{\text {diag }}$ where $M_{R R}$ is in the diagonal basis of $m_{L R}$. We follow this representation in the present calculation.

In some Grand Unified Theory such as $S O(10)$ GUT , the possible structure[16] of $m_{L R}=\operatorname{diag}\left(\lambda^{m}, \lambda^{n}, 1\right) v$, where $v$ is the overall scale factor representing electroweak vacuum expectation values. In the present calculation we take $\lambda=0.3$ and $v=174 G e V$. We consider two choices of $(m, n)$ pair: case(i) $(m, n) \equiv(6,2)$ for charged lepton and (ii) $(m, n) \equiv(8,4)$ for up-quark mass matrices representing the Dirac neutrino mass matrix.

As a representative example, we consider normal hierarchical mass model and the corresponding heavy right-handed neutrino mass matrix and light lefthanded neutrino mass matrices are[1,2] collected from Appendix B:

Normal Hierarchical Type3(NHT3):

$$
\begin{gathered}
M_{R R}=\left(\begin{array}{ccc}
\lambda^{2 m-1} & \lambda^{m+n-1} & \lambda^{m-1} \\
\lambda^{m+n-1} & \lambda^{m+n-2} & 0 \\
\lambda^{m-1} & 0 & 1
\end{array}\right) v_{0}, \\
-m_{L L}^{I}=\left(\begin{array}{ccc}
-\lambda^{4} & \lambda & \lambda^{3} \\
\lambda & 1-\lambda & -1 \\
\lambda^{3} & -1 & 1-\lambda^{3}
\end{array}\right) m_{0},
\end{gathered}
$$

where we take the input values $\lambda=0.3, m_{0}=0.03 \mathrm{eV}$ and $v_{0}=1.01 \times 10^{15} \mathrm{GeV}$.

The numerical calculations are carried out for two choices of diagonal structure of Dirac neutrino mass matrix: case(i) Dirac neutrino mass matrix taken as charged lepton mass matrix and case(ii) Dirac neutrino mass matrix taken as up-quark mass matrix. Within the type-I seesaw framework, the left-handed neutrino mass matrix $m_{L L}^{I}$ leads to correct neutrino mass parameters and mixing angles consistent with recent data[1,2]:

$$
\begin{gathered}
\Delta m_{21}^{2}=9.04 \times 10^{-5} \mathrm{eV}^{2}, \Delta m_{23}^{2}=3.01 \times 10^{-3} \mathrm{eV}^{2} \\
\tan ^{2} \theta_{12}=0.55, \sin ^{2} 2 \theta_{23}=0.98, \sin \theta_{13}=0.074 .
\end{gathered}
$$

In type-II seesaw scenario, one has to take care of the non-canonical term $m_{L L}^{I I}$. In order to maintain the existing good predictions of $m_{L L}^{I}$, one has to use the freedom in choosing $\gamma$. We make a search programme[2] for finding the value of the 'minimum departure' of $\gamma$ from the canonical value of one, i.e., $\gamma<1.0$, in which the good prediction of neutrino masses and mixing parameters can again be restored in $m_{L L}$. In other words, such 'least value' of $\gamma$ is just enough to suppress the perturbative effect due to type-II seesaw formula. The value of $\gamma=0.007$ is extracted for NHT3 mass model. The values of $\gamma$ in case(i) and case(ii) for other neutrino mass models given in Appendix B, are presented 
in Tables 2-3, along with the corresponding predictions of neutrino oscillation parameters. Higher the values of $\gamma$, more the stability of the neutrino mass model in question. Following the results of calculation in Tables 1-3. it has been observed that InvT2A model with $\gamma=0.1$ gives most favourable choice of nature, followed by NHT3 and InvT2B with $\gamma \sim 10^{-2}$. However, InvT2A model appears to be unstable under radiative corrections in MSSM. Now we are left with NHT3 and InvT2B as favourable candidates which are generally stable under radiative corrections in MSSM.

For further discrimination of the neutrino mass models, particularly between NHT3 and InvT2B, we now consider baryon asymmetry estimation within the type-II seesaw formalism as an extra criteria and this will be carried out in the following section as the main thrust of the present invistigation.

\section{Type-II seesaw mechanism and the estimation of baryon asymmetry}

For our calculation of lepton asymmetry we consider the model[5] where the asymmetric decay of the lightest of the heavy right-handed Majorana neutrinos, is assumed. In this model the physical Majorana neutrino $N_{R}$ can decay into two modes:

$$
\begin{aligned}
& N_{R} \rightarrow l_{L}+\bar{\phi} \\
& \quad \rightarrow \bar{l}_{L}+\phi
\end{aligned}
$$

where $l_{L}$ is the lepton and $\bar{l}_{L}$ is the antilepton. For CP-violating decay through the one-loop radiative correction by Higgs particle, the branching ratio for these two decay modes is likely to be different. The CP-asymmetry which is caused by the intereference of tree level with one-loop corrections for the decays of lightest of heavy right-handed Majorana neutrino $N_{1}$ is defined as[5,17]

$$
\epsilon=\frac{\Gamma-\bar{\Gamma}}{\Gamma+\bar{\Gamma}}
$$

where, $\Gamma=\Gamma\left(N_{1} \rightarrow l_{L} \bar{\phi}\right)$ and $\bar{\Gamma}=\Gamma\left(N_{1} \rightarrow \overline{l_{L}} \phi\right)$ are the decay rates. Considering hierarchical structure of heavy Majorana neutrinos a perturbative calculation from the interference between tree level and vertex plus self-energy diagrams, gives[18] the lepton asymmetry $\epsilon^{I}$ in terms of light neutrino mass matrix $m_{L L}^{I}$ as :

$$
\epsilon^{I}=-\frac{3 M_{1}}{16 \pi v^{2}} \frac{\operatorname{Im}\left[\left(h^{*} m_{L L}^{I} h^{\dagger}\right)_{11}\right]}{\left(h h^{\dagger}\right)_{11}} .
$$

This accounts for the type-I contribution. Again due to the presence of Higgs triplet as a virtual particle in the decay of $N_{1}$, we have the additional contribution [18] (type-II contribution) to lepton asymmetry $\epsilon^{I I}$ as:

$$
\epsilon^{I I}=-\frac{3 M_{1}}{16 \pi v^{2}} \frac{\operatorname{Im}\left[\left(h^{*} m_{L L}^{I I} h^{\dagger}\right)_{11}\right]}{\left(h h^{\dagger}\right)_{11}} .
$$


For quasi-degenerate spectrum i.e., for $M_{1} \simeq M_{2}<M_{3}$ the asymmetry is largely affected by a resonance factor $[19,20] R=\left|M_{1}\right| / 2\left(\left|M_{2}\right|-\left|M_{1}\right|\right)$. In such situation , the lepton asymmetry is modified $[19,20]$ to:

$$
\epsilon=-\frac{M_{2}}{8 \pi v^{2}} \frac{\operatorname{Im}\left[\left(h^{*} m_{L L} h^{\dagger}\right)_{11}\right]}{\left(h h^{\dagger}\right)_{11}} R
$$

The total asymmetry $\epsilon_{1}$ is the sum of $\epsilon^{I}$ and $\epsilon^{I I}$.

The sphaleron process convert $[4,21]$ this lepton asymmetry to baryon asymmetry. The baryon asymmetry $Y_{B}^{S M}$ is expressed in terms of the dilution factor $\kappa_{1}$ and lepton asymmetry $\epsilon_{1}$ as $[22]$

$$
Y_{B}^{S M} \equiv\left(\frac{n_{B}}{n_{\gamma}}\right)^{S M} \simeq-1.08 \times 10^{-2} \kappa_{1} \epsilon_{1} .
$$

For our calculation of baryon asymmetry we use the above expression.

How much the produced asymmetry is washed out is described by Boltzmann Equation and the solution can be described by a parameter $\kappa_{1}$ known as dilution factor[14,23]:

$$
\kappa_{1} \simeq \begin{cases}\frac{0.3}{K(\ln K)^{0.6}} & \text { if } 10 \leq K \leq 10^{6} \\ \frac{1}{2 \sqrt{K^{2}+9}} & \text { if } 0 \leq K \leq 10\end{cases}
$$

Where $K=\tilde{m}_{1} / m^{*}$ with $\tilde{m}_{1}=\frac{\left(h h^{\dagger}\right)_{11} v^{2}}{M_{1}}$ is the effective neutrino mass , $v$ the electroweak scale, $M_{1}$ the mass of $N_{1}^{1}, h$ the matrix for the neutrino Yukawa couplings. And $m^{*}=\frac{16 \pi^{\frac{5}{2}}}{3 \sqrt{5}} g_{*}^{\frac{1}{2}} \frac{v^{2}}{M_{p l}} \simeq 1.08 \times 10^{-3} \mathrm{eV}$ is known as equilibrium neutrino mass[24] with $g^{*}=106.75, M_{p l}=1.2 \times 10^{19} \mathrm{GeV}$. Thus, for $\tilde{m}_{1}<m^{*}$, the heavy right-handed neutrino will satisfy the out-of-equilibrium condition. For our calculation we consider this condition also.

\subsection{Numerical Calculation and results}

For our calculation of lepton asymmetry, we choose a basis $U_{R}$ where $M_{R R}^{\text {diag }}=$ $U_{R}^{T} M_{R R} U_{R}=\operatorname{diag}\left(M_{1}, M_{2}, M_{3}\right)$ with real and positive eigenvalues[15,25]. We transform $m_{L R}=\operatorname{diag}\left(\lambda^{m}, \lambda^{n}, 1\right) v$ to the $U_{R}$ basis by $m_{L R} \rightarrow m_{L R}^{\prime} U_{R}$. In the prime basis the Yukawa coupling becomes $h=\left(m_{L R} U_{R}\right) / v$. For demonstration as an example, we consider here the normal hierarchical mass model(NHT3) described in section 2. The corresponding heavy right-handed neutrino mass matrix and light left-handed neutrino mass matrices are given in eq.(4) and eq.(5).

For case(i): charged lepton mass matrix, $m_{L R}=m_{E}=\operatorname{diag}\left(\lambda^{6}, \lambda^{2}, 1\right) v$, $(m, n)=(6,2), v_{0}=1.01 \times 10^{15} \mathrm{GeV}, m_{0}=0.03 \mathrm{eV}, \gamma=0.007$. For the above input values the three heavy masses are $M_{R R}^{\operatorname{diag}}=\operatorname{diag}\left(4.28 \times 10^{9}, 1.16 \times 10^{10}, 3.84 \times\right.$ 
$\left.10^{13}\right)$ in GeV. From the construction of ' $h$ ', we have $\left(h h^{\dagger}\right)_{11}=5.31 \times 10^{-7}$, $\operatorname{Im}\left(h^{*} m_{L L}^{I} h^{\dagger}\right)_{11}=2.45 \times 10^{-18}, \operatorname{Im}\left(h^{*} m_{L L}^{I I} h^{\dagger}\right)_{11}=2.52 \times 10^{-24}, K=0.23$ and $\kappa_{1}=0.17$. These leads to lepton asymmetry $\epsilon^{I}=5.92 \times 10^{-7}$ and $\epsilon^{I I}=6.09 \times 10^{-13}$ resulting $\epsilon_{1}=\epsilon^{I}+\epsilon^{I I}=5.92 \times 10^{-7}$. The corresponding baryon asymmetry is $Y_{B}=-1.08 \times 10^{-2} \kappa_{1} \epsilon_{1}=1.08 \times 10^{-9}$.

For all other models including the normal hierarchy case(ii) we follow the same procedure to calculate the lepton and baryon asymmetry. For case (ii) the Dirac neutrino mass matrix is taken as the up quark mass matrix i.e., $m_{L R}=$ $\operatorname{diag}\left(\lambda^{8}, \lambda^{4}, 1\right) v$. As quasi-degenerate pattern of heavy masses appears in Inverted hierarchy type2B (InvT2B), so for this model we consider the resonance enhancement factor $R$, and a different expression[19] $Y_{B}=-2.16 \times 10^{-2} \kappa_{1} \epsilon_{1}$ is used for the estimation of baryon asymmetry. The estimated values of lepton and baryon asymmetry for all the models in Appendix B are collected in tables 6-8. As emphasised earlier, we use the only parameters fixed at seesaw stage in section 2 for estimating baryon asymmetry as an additional criteria for discriminating neutrino mass models.

\section{Summary and Discussion}

To summarise, we have considered various neutrino mass models: degenerate models (DegT1A, DegT1B, DegT1C), inverted hierarchical (InvT2A, InvT2B) and normal hierarchical( NHT3). We have estimated the contributions of type-I (canonical) and type-II ( non-canonical) seesaw mass term towards baryogenesis. Following the predictions of $S O(10)$ grand unified theory, we estimate the baryon asymmetry for two choices of Dirac neutrino mass matrix: case(i) Dirac neutrino mass matrix taken as charged lepton mass matrix and case(ii) Dirac neutrino mass matrix taken as up quark mass matrix. For specific values of the discriminator ' $\gamma$ ' extracted from type-II seesaw formula[2], we observe that the baryon asymmetry is dominated by type-I term only.

All the eigenvalues of $M_{R R}$ are collected in Table-4, and the mass parameter $\tilde{m}_{1}$, dilution factor $\kappa_{1}$ are presented in Table- 5 . From Table- 5 it is clear that only inverted hierarchical model type2B (InvT2B) and normal hierarchical model type-3 (NHT3) satisfy the out-of-equilibrium decay condition. In these two models, the effective neutrino mass is less than the equilibrium neutrino mass $m^{*}\left(\simeq 1.08 \times 10^{-3}\right)$ i.e., $\tilde{m}_{1}<m^{*}$.

In Tables 6-8 we present the estimations of lepton and baryon asymmetry. We present the numerical predictions of the neutrino mass-squared differences and three mixing angles in type-I and type-II seesaw framework[2] in Tables 1-3. Only two models i.e., inverted hierarchical model type2A (InvT2A) and normal hierarchical model type 3 (NRT3) satisfy all the neutrino oscillation parameters (Tables 1-3). However, the inverted hierarchical Type-2A(InvT2A) model is not stable under radiative corrections in MSSM, whereas normal hierarchical type 3 (NHT3) is stable.

If we consider the observed baryon asymmetry[26] $Y_{B}=\left(6.1_{-0.2}^{-0.3}\right) \times 10^{-10}$, a competitive nature appears for both Degenerate type-1 model (DegT1A) and Normal hierarchical type3(NHT3). But considering the mixing parameters and 
stability condition, one can easily rule out the degenerate type 1A (DegT1A) model. For NHT3 the mass of $M_{1}$ is also within the Ibarra-Davidson bound [27]. Considering the above results we have found that in type-II scenario normal hierarchical type 3(NHT3) model is the most favourable choice of nature.

The present calculation may also be useful to discriminate the choices of Dirac neutrino mass matrix. The charged lepton, up-quark and down-quark approximation of Dirac neutrino mass matrix may not be the right choice of nature. Once a neutrino mass model is fixed by experiment, then one can search for the possible structure of Dirac neutrino mass matrix by constraining observed baryon asymmetry to such model[28]. 
Table-1: Predicted values of the solar and atmospheric neutrino mass-squared differences and three mixing parameters calculated from $m_{L L}^{I}$ derived from typeI seesaw formula in the Appendix-B.

\begin{tabular}{cccccc}
\hline Type & $\Delta m_{21}^{2}\left[10^{-5} \mathrm{eV}^{2}\right]$ & $\Delta m_{23}^{2}\left[10^{-3} \mathrm{eV}^{2}\right]$ & $\tan ^{2} \theta_{12}$ & $\sin ^{2} 2 \theta_{23}$ & $\sin \theta_{13}$ \\
\hline DegT1A & 8.80 & 2.83 & 0.98 & 1.0 & 0.0 \\
DegT1B & 7.91 & 2.50 & 0.27 & 1.0 & 0.0 \\
DegT1C & 7.91 & 2.50 & 0.27 & 1.0 & 0.0 \\
InvT2A & 8.36 & 2.50 & 0.44 & 1.0 & 0.0 \\
InvT2B & 9.30 & 2.50 & 0.98 & 1.0 & 0.0 \\
NHT3 & 9.04 & 3.01 & 0.55 & 0.98 & 0.074 \\
\hline
\end{tabular}

Table-2:Predicted values of the solar and atmospheric neutrino mass-squared differences and three mixing parameters along with the values of $\gamma$, extracted from $m_{L L}$ in type-II seesaw formula for case (i).

\begin{tabular}{ccccccc}
\hline Type & $\gamma$ & $\triangle m_{21}^{2}\left[10^{-5} \mathrm{eV}^{2}\right]$ & $\triangle m_{23}^{2}\left[10^{-3} \mathrm{eV}^{2}\right]$ & $\tan ^{2} \theta_{12}$ & $\sin ^{2} 2 \theta_{23}$ & $\sin \theta_{13}$ \\
\hline DegT1A & $10^{-5}$ & 8.45 & 2.73 & 0.98 & 1.00 & 0.0 \\
DegT1B & $10^{-4}$ & 7.97 & 2.30 & 0.28 & 1.00 & 0.0 \\
DegT1CC & $10^{-5}$ & 7.93 & 2.47 & 0.27 & 1.00 & 0.0 \\
InvT2A & 0.1 & 8.20 & 2.50 & 0.49 & 1.00 & 0.0 \\
InvT2B & 0.009 & 9.40 & 2.40 & 0.98 & 1.00 & 0.01 \\
NHT3 & 0.007 & 9.41 & 2.98 & 0.54 & 0.98 & 0.09 \\
\hline
\end{tabular}

Table-3:Predicted values of solar and atmospheric neutrino mass-squared differences, and three mixing parameters along with the values of $\gamma$, extracted from $m_{L L}$ in type-II seesaw formula for case (ii).

\begin{tabular}{ccccccc}
\hline Type & $\gamma$ & $\triangle m_{21}^{2}\left[10^{-5} \mathrm{eV}^{2}\right]$ & $\triangle m_{23}^{2}\left[10^{-3} \mathrm{eV}^{2}\right]$ & $\tan ^{2} \theta_{12}$ & $\sin ^{2} 2 \theta_{23}$ & $\sin \theta_{13}$ \\
\hline DegT1A & $10^{-5}$ & 8.56 & 2.74 & 0.98 & 1.00 & 0.0 \\
DegT1B & $10^{-4}$ & 7.69 & 2.30 & 0.27 & 1.00 & 0.0 \\
DegT1CC & $10^{-5}$ & 7.69 & 2.54 & 0.29 & 1.00 & 0.0 \\
InvT2A & 0.1 & 8.3 & 2.5 & 0.47 & 1.00 & 0.0 \\
InvT2B & 0.02 & 9.40 & 2.40 & 0.98 & 1.00 & 0.0 \\
NHT3 & 0.007 & 9.18 & 2.80 & 0.55 & 0.98 & 0.07 \\
\hline
\end{tabular}


Table-4: The three right-handed Majorana neutrino masses in GeV for both case (i) and case (ii). The expressions of $M_{R R}$ are collected from Appendix-B.

\begin{tabular}{cc|c}
\hline Type & Case(i): $\left|M_{j}\right|$ & Case(ii): $\left|M_{j}\right|$ \\
\hline DegT1A & $4.28 \times 10^{9}, 1.16 \times 10^{10}, 3.84 \times 10^{13}$ & $3.47 \times 10^{7}, 9.42 \times 10^{7}, 3.81 \times 10^{13}$ \\
DegT1B & $4.05 \times 10^{7}, 6.16 \times 10^{11}, 7.60 \times^{13}$ & $3.28 \times 10^{5}, 4.98 \times 10^{9}, 7.60 \times 10^{13}$ \\
DegT1C & $4.05 \times 10^{7}, 6.69 \times 10^{12}, 6.69 \times 10^{12}$ & $3.28 \times 10^{5}, 4.85 \times 10^{11}, 7.81 \times 10^{11}$ \\
InvT2A & $3.28 \times 10^{8}, 9.70 \times 10^{12}, 6.79 \times 10^{16}$ & $2.64 \times 10^{6}, 7.92 \times 10^{10}, 6.70 \times 10^{16}$ \\
InvT2B & $5.6527 \times 10^{10}, 5.6532 \times 10^{10}, 5.38 \times 10^{16}$ & $4.5971 \times 10^{8}, 4.5974 \times 10^{8}, 5.34 \times 10^{16}$ \\
NHT3 & $6.51 \times 10^{10}, 7.97 \times 10^{11}, 1.01 \times 10^{15}$ & $5.27 \times 10^{8}, 6.45 \times 10^{9}, 1.01 \times 10^{15}$ \\
\hline
\end{tabular}

Table-5 :Estimation of the values of effective mass parameter $\tilde{m}_{1}$ in $\mathrm{eV}$, and dilution factor $\kappa_{1}$.

\begin{tabular}{cccc|ccc}
\hline & For & case $(\mathrm{i})$ & & For & case(ii) & \\
\hline Type & $\tilde{m}_{1}$ & $K$ & $\kappa_{1}$ & $\tilde{m}_{1}$ & $K$ & $\kappa_{1}$ \\
\hline DegT1A & $3.76 \times 10^{-3}$ & 3.48 & 0.11 & $3.74 \times 10^{-3}$ & 3.46 & 0.11 \\
DegT1B & 0.40 & 370 & $2.79 \times 10^{-4}$ & 0.40 & 370 & $2.79 \times 10^{-4}$ \\
DegT1C & 0.40 & 370 & $2.79 \times 10^{-4}$ & 0.40 & 370 & $2.79 \times 10^{-4}$ \\
Inv2A & 0.05 & 46.3 & $2.9 \times 10^{-3}$ & 0.05 & 46.3 & $2.9 \times 10^{-3}$ \\
InvT2B & $2.85 \times 10^{-4}$ & 0.26 & 0.17 & $2.83 \times 10^{-4}$ & 0.26 & 0.17 \\
NHT3 & $2.47 \times 10^{-4}$ & 0.23 & 0.17 & $2.47 \times 10^{-4}$ & 0.23 & 0.17 \\
\hline
\end{tabular}

Table 6: Calculation of lepton asymmetry $\epsilon^{I}$ and $\epsilon^{I I}$ for case(i) for respective neutrino mass models given in Appendix-B.

\begin{tabular}{ccc|c}
\hline Type & $\epsilon^{I}$ & $\epsilon^{I I}$ & $\epsilon_{1}=\epsilon^{I}+\epsilon^{I I}$ \\
\hline DegT1A & $2.10 \times 10^{-6}$ & $1.19 \times 10^{-15}$ & $2.10 \times 10^{-6}$ \\
DegT1B & $2.66 \times 10^{-18}$ & $3.88 \times 10^{-27}$ & $2.66 \times 10^{-18}$ \\
DegT1C & $1.74 \times 10^{-18}$ & $2.24 \times 10^{-25}$ & $1.74 \times 10^{-18}$ \\
InvT2A & $1.59 \times 10^{-14}$ & $5.30 \times 10^{-26}$ & $1.59 \times 10^{-14}$ \\
InvT2B & $1.47 \times 10^{-2}$ & $2.52 \times 10^{-13}$ & $1.47 \times 10^{-2}$ \\
NRT3 & $5.92 \times 10^{-7}$ & $6.09 \times 10^{-13}$ & $5.92 \times 10^{-7}$ \\
\hline
\end{tabular}


Table 7: Calculation of lepton asymmetry $\epsilon^{I}$ and $\epsilon^{I I}$ for case(ii) for respective neutrino mass models given in Appendix-B.

\begin{tabular}{ccc|c}
\hline Type & $\epsilon^{I}$ & $\epsilon^{I I}$ & $\epsilon_{1}=\epsilon^{I}+\epsilon^{I I}$ \\
\hline DegT1A & $1.71 \times 10^{-8}$ & $7.98 \times 10^{-20}$ & $1.17 \times 10^{-8}$ \\
DegT1B & $2.16 \times 10^{-20}$ & $2.54 \times 10^{-31}$ & $2.16 \times 10^{-20}$ \\
DegT1C & $1.69 \times 10^{-20}$ & $1.43 \times 10^{-26}$ & $1.69 \times 10^{-20}$ \\
InvT2A & $1.27 \times 10^{-16}$ & $2.46 \times 10^{-28}$ & $1.27 \times 10^{-16}$ \\
InvT2B & $1.62 \times 10^{-4}$ & $5.08 \times 10^{-17}$ & $1.62 \times 10^{-4}$ \\
NRT3 & $4.78 \times 10^{-9}$ & $4.90 \times 10^{-17}$ & $4.78 \times 10^{-9}$ \\
\hline
\end{tabular}

Table-8: Calculation of baryon asymmetry $Y_{B}$ for respective neutrino mass models given in Appendix-B.

\begin{tabular}{cc|c}
\hline Type & $Y_{B}^{S M}$ case $(i)$ & $Y_{B}^{S M}$ case $(i i)$ \\
\hline DegT1A & $2.49 \times 10^{-9}$ & $2.03 \times 10^{-11}$ \\
DegT1B & $8.00 \times 10^{-24}$ & $6.50 \times 10^{-26}$ \\
DegT1C & $5.20 \times 10^{-24}$ & $5.10 \times 10^{-26}$ \\
InvT2A & $4.97 \times 10^{-19}$ & $3.98 \times 10^{-21}$ \\
InvT2B & $5.40 \times 10^{-5}$ & $5.94 \times 10^{-7}$ \\
NHT3 & $1.08 \times 10^{-9}$ & $8.80 \times 10^{-12}$ \\
\hline
\end{tabular}




\section{Appendix A}

We list here the zeroth-order left-handed Majorana neutrino mass matrices[2,13] with texture zeros, $m_{L L}^{I}$, corresponding to three models of neutrinos, viz., degenerate (DegT1A, DegT1B, DegT1C), inverted hierarchical (InvT2A, InvT2B) and normal hierarchical (NHT3).

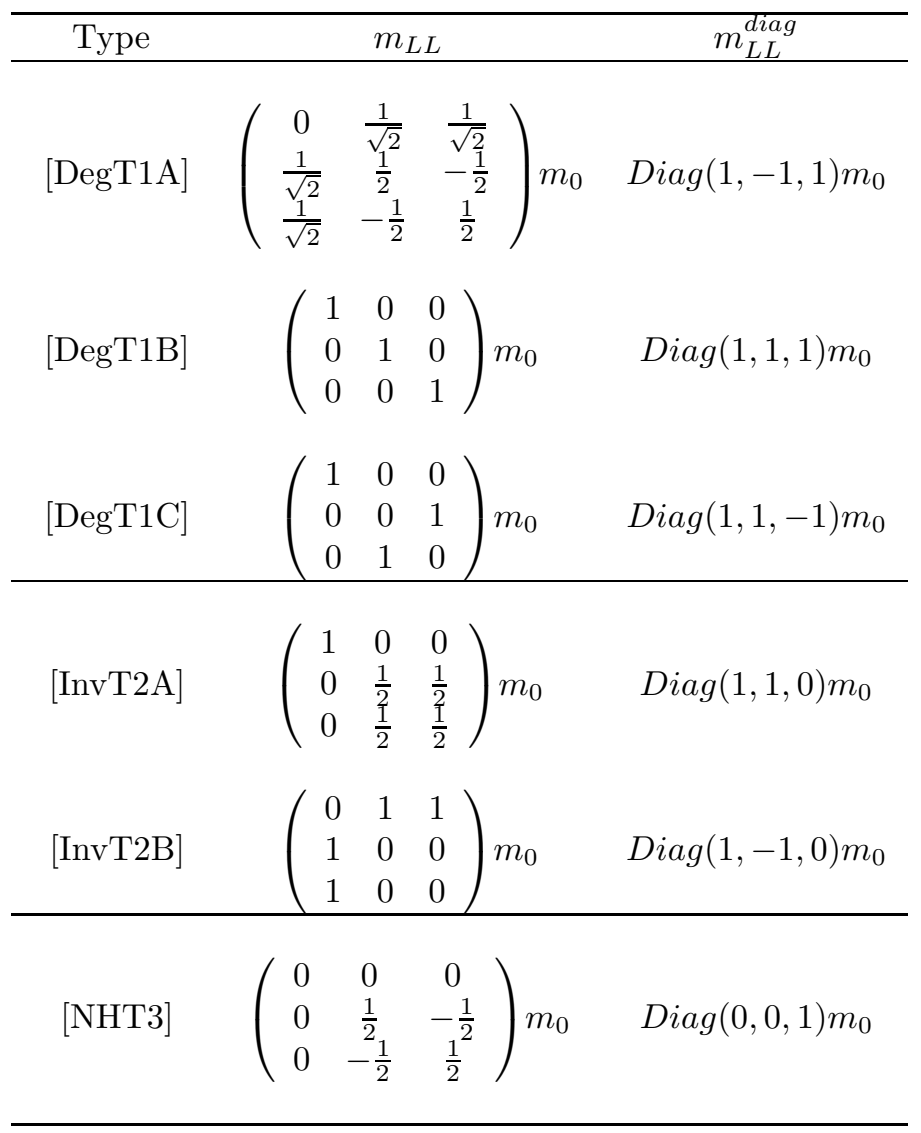




\section{Appendix B}

Here we collect $[1,2]$ the various right handed Majorana mass matrices $M_{R R}$ and the light left handed neutrino mass matrix $m_{L L}^{I}$ along with the input values of the parameters.

$\underline{\text { Degenerate Type1A(DegT1A): }}$

$$
\begin{aligned}
\mathbf{M}_{\mathbf{R R}}= & \left(\begin{array}{ccc}
-2 \delta_{2} \lambda^{2 m} & \left(\frac{1}{\sqrt{2}}+\delta_{1}\right) \lambda^{m+n} & \left(\frac{1}{\sqrt{2}}+\delta_{1}\right) \lambda^{m} \\
\left(\frac{1}{\sqrt{2}}+\delta_{1}\right) \lambda^{m+n} & \left(1 / 2+\delta_{1}-\delta_{2}\right) \lambda^{2 n} & \left(-1 / 2+\delta_{1}-\delta_{2}\right) \lambda^{n} \\
\left(\frac{1}{\sqrt{2}}+\delta_{1}\right) \lambda^{m} & \left(-1 / 2+\delta_{1}-\delta_{2}\right) \lambda^{n} & \left(1 / 2+\delta_{1}-\delta_{2}\right)
\end{array}\right) v_{0} \\
& -\mathbf{m}_{\mathbf{L} \mathbf{L}}^{\mathbf{I}}=\left(\begin{array}{ccc}
\left(-2 \delta_{1}+2 \delta_{2}\right) & \left(\frac{1}{\sqrt{2}}-\delta_{1}\right) & \left(\frac{1}{\sqrt{2}}-\delta_{1}\right) \\
\left(\frac{1}{\sqrt{2}}-\delta_{1}\right) & \left(1 / 2+\delta_{2}\right) & \left(-1 / 2+\delta_{2}\right) \\
\left(\frac{1}{\sqrt{2}}-\delta_{1}\right) & \left(-1 / 2+\delta_{2}\right) & \left(1 / 2+\delta_{2}\right)
\end{array}\right) m_{0}
\end{aligned}
$$

Here, $\delta_{1}=0.0061875, \delta_{2}=0.0031625, \lambda=0.3, m_{0}=0.4 \mathrm{eV}$ and $v_{0}=7.57 \times$ $10^{13} \mathrm{GeV}, \gamma=10^{-5}$.

Degenerate Type1B(DegT1B):

$$
\begin{gathered}
\mathbf{M}_{\mathbf{R R}}=\left(\begin{array}{ccc}
\left(1+2 \delta_{1}+2 \delta_{2}\right) \lambda^{2 m} & \delta_{1} \lambda^{m+n} & \delta_{1} \lambda^{m} \\
\delta_{1} \lambda^{m+n} & \left(1+\delta_{2} \lambda^{2 n}\right. & \delta_{2} \lambda^{n} \\
\delta_{1} \lambda^{m} & \delta_{2} \lambda^{n} & \left(1+\delta_{2}\right)
\end{array}\right) v_{0} \\
-\mathbf{m}_{\mathbf{L L}}^{\mathbf{I}}=\left(\begin{array}{ccc}
\left(1-2 \delta_{1}-2 \delta_{2}\right) & -\delta_{1} & -\delta_{1} \\
-\delta_{1} & \left(1-\delta_{2}\right) & -\delta_{2} \\
-\delta_{1} & -\delta_{2} & \left(1-\delta_{2}\right)
\end{array}\right) m_{0}
\end{gathered}
$$

Here, $\delta_{1}=7.2 \times 10^{-5}, \delta_{2}=3.9 \times 10^{-3}, \lambda=0.3, m_{0}=0.4 \mathrm{eV}$ and $v_{0}=7.57 \times$ $10^{13} \mathrm{GeV}, \gamma=10^{-4}$.

$\underline{\text { Degenerate Type1C(DegT1C): }}$

$$
\begin{gathered}
\mathbf{M}_{\mathbf{R R}}=\left(\begin{array}{ccc}
\left(1+2 \delta_{1}+2 \delta_{2}\right) \lambda^{2 m} & \delta_{1} \lambda^{m+n} & \delta_{1} \lambda^{m} \\
\delta_{1} \lambda^{m+n} & \delta_{2} \lambda^{2 n} & \left(1+\delta_{2}\right) \lambda^{n} \\
\delta_{1} \lambda^{m} & \left(1+\delta_{2}\right) \lambda^{n} & \delta_{2}
\end{array}\right) v_{0} \\
-\mathbf{m}_{\mathbf{L L}}^{\mathbf{I}}=\left(\begin{array}{ccc}
\left(1-2 \delta_{1}-2 \delta_{2}\right) & -\delta_{1} & -\delta_{1} \\
-\delta_{1} & -\delta_{2} & \left(1-\delta_{2}\right) \\
-\delta_{1} & \left(1-\delta_{2}\right) & -\delta_{2}
\end{array}\right) m_{0}
\end{gathered}
$$


Here, $\delta_{1}=7.2 \times 10^{-5}, \delta_{2}=3.9 \times 10^{-3}, \lambda=0.3, m_{0}=0.4 \mathrm{eV}$ and $v_{0}=7.57 \times$ $10^{13} \mathrm{GeV}, \gamma=10^{-5}$.

$\underline{\text { Inverted Hierarchical Type2A(InvT2A): }}$

$$
\begin{aligned}
\mathbf{M}_{\mathbf{R R}}= & \left(\begin{array}{ccc}
\eta(1+2 \epsilon) \lambda^{2 m} & \eta \epsilon \lambda^{m+n} & \eta \epsilon \lambda^{m} \\
\eta \epsilon \lambda^{m+n} & 1 / 2 \lambda^{2 n} & -(1 / 2-\eta) \lambda^{n} \\
\eta \epsilon \lambda^{m} & -(1 / 2-\eta) \lambda^{n} & 1 / 2
\end{array}\right) \frac{v_{0}}{\eta} \\
& -\mathbf{m}_{\mathbf{L L}}^{\mathbf{I}}=\left(\begin{array}{ccc}
(1-2 \epsilon) & -\epsilon & -\epsilon \\
-\epsilon & \frac{1}{2} & \left(\frac{1}{2}-\eta\right) \\
-\epsilon & \left(\frac{1}{2}-\eta\right) & \frac{1}{2}
\end{array}\right) m_{0}
\end{aligned}
$$

Here, $\eta=0.0045, \epsilon=0.0055, \lambda=0.3, m_{0}=0.05 \mathrm{eV}$ and $v_{0}=6.06 \times 10^{13} \mathrm{GeV}$, $\gamma=0.1$.

$\underline{\text { Inverted Hierarchical Type2B(InvT2B): }}$

$$
\begin{gathered}
\mathbf{M}_{\mathbf{R R}}=\left(\begin{array}{ccc}
\lambda^{2 m+7} & \lambda^{m+n+4} & \lambda^{m+4} \\
\lambda^{m+n+4} & \lambda^{2 n} & -\lambda^{n} \\
\lambda^{m+4} & -\lambda^{n} & 1
\end{array}\right) v_{0} \\
-\mathbf{m}_{\mathbf{L L}}^{\mathbf{I}}=\left(\begin{array}{ccc}
0 & 1 & 1 \\
1 & -\left(\lambda^{3}-\lambda^{4}\right) / 2 & -\left(\lambda^{3}+\lambda^{4}\right) / 2 \\
1 & -\left(\lambda^{3}+\lambda^{4}\right) / 2 & -\left(\lambda^{3}-\lambda^{4}\right) / 2
\end{array}\right) m_{0}
\end{gathered}
$$

Here, $\lambda=0.3, m_{0}=0.035 \mathrm{eV}$ and $v_{0}=5.34 \times 10^{16} \mathrm{GeV}$. For case(i) $\gamma=0.009$ and for case(ii) $\gamma=0.02$.

Normal Hierarchical Type3(NHT3):

$$
\begin{gathered}
\mathbf{M}_{\mathbf{R R}}=\left(\begin{array}{ccc}
\lambda^{2 m-1} & \lambda^{m+n-1} & \lambda^{m-1} \\
\lambda^{m+n-1} & \lambda^{m+n-2} & 0 \\
\lambda^{m-1} & 0 & 1
\end{array}\right) v_{0} \\
-\mathbf{m}_{\mathbf{L L}}^{\mathbf{I}}=\left(\begin{array}{ccc}
-\lambda^{4} & \lambda & \lambda^{3} \\
\lambda & 1-\lambda & -1 \\
\lambda^{3} & -1 & 1-\lambda^{3}
\end{array}\right) m_{0}
\end{gathered}
$$

Here, $\lambda=0.3, m_{0}=0.03 \mathrm{eV}$ and $v_{0}=1.01 \times 10^{15} \mathrm{GeV}, \gamma=0.007$.

\section{Acknowledgements}

One of us (AKS) would like to thank UGC(NER), India, for awarding a fellowship under FIP programme, Xth plan. 


\section{References}

[1] N. Nimai Singh and M. Patgiri, IJMP A17, 3629 (2002); M. Patgiri and N. N. Singh, IJMP A18, 443 (2003).

[2] N.Nimai Singh, M.Patgiri and M.K.Das, Pramana-Journal of Physics, 66(2) (2006)361.

[3] M.Gell-Mann,P.Ramond and R.Slansky in Supergravity,Proceeding of the Workshop,StonyBrook,NewYork,1979,Edited by P.Van Nieumenhuizen and D.Freedman(North-Holland,Amsterdam1979),T.Yanagida,KEKLectures1979 (unpublished); R.N.Mahapatra and G.Senjanovic,Phy.Rev.Lett.44,912(1980).

[4] V.A.Kuzmin, V.A.Rubakov, M.E.Shaposhnikov, Phy.Lett.B155(1985)36.

[5] M.Fukugita and T.Yanagida, Phys. Lett.B174(1986)45; M. A. Luty, Phy.Rev D45(1992), U Sarkar hep-ph/9810247.

[6] N. S Manton, Phys. Rev.D28 (1983)2019; F. R. Klinkhamer and N. S. Manton, Phys. Rev.D30 (1984)2212.

[7] A.D.Sakharov, JETP Lett.5(1967)24.

[8] G.Panotopoulos, hep-ph/0606127.

[9] A.K. Sarma, H.Z. Devi and N. Nimai Singh hep-ph/0604040.

[10] R.N.Mohapatra and G. Senjanovic, Phys.Rev.D23,165(1981); G. Lazarides, Q.Shafi, C. Wetterich, Nucl.Phys. B181, 287(1981); C. Wetterich, Nucl.Phys.B187,343(1981).

[11] B. Bajc, G. Senjanovic and F. Vissani, Phys. Rev.Lett 90(2003)051802 hep-ph/0110310 ; H. S. Goh, R. N. Mahapatra and S.P. Ng, hep-ph/0303055

[12] C. S.Aulakh, B. Bajc, A.Melfo, G.Senjanovic and F. Vissani, hep-ph/0306242

[13] G. Altarelli and F. Feruglio, Phys. Rep. 320(1999)295, hep-ph/9905536

[14] A. S. Joshipura, E. A. Paschos, W. Rodejohann, JHEP 0108 (2001); Nucl. Phys. B611, 227 (2001).

[15] E. K. Akhmedov, M. Frigerio and A. Y. Smirnov, JHEP 0309(2003) 021 hep-ph/0305322.

[16] P. M. Fishbane and P. Kaus, J. Phys.G:Nucl. Part. Phys.25(1999)16291640 .

[17] E. W. Kolb, M. S. Turner, The Early Universe, Addision- Wesely, NewYork (1990). 
[18] N. Sahu and S. U. Sankar, Phys. Rev.D71(2005)013006, hep-ph/0406065 T. Hambye and G. Senjanovic, Phys. Lett. B582, (2004)73, hep-ph/0307237; S. Antush and S. F. King, Phys. Lett. B597,(2004),199 hep-ph/0405093.

[19] Carl H. Albright, S. M. Barr, Phys.Rev. D70, (2004)033013, hep-ph/0404095.

[20] T.Hambye, ICTP Summer School Lecture Notes(2004).

[21] J. A. Harvey and M. S. Turner, Phys. Rev. D42, 3344 (1990), U Sarkar hep-ph/9810247.

[22] L. Covi, E. Roulet and F. Vissani, Phys. Lett. B384 (1996) 169 hep-ph/9605319.

[23] P. Di Bari, hep-ph/0406115 hep-ph/0211175.

[24] W. Buchmuller, hep-ph/0306047.

[25] K.S.Babu , A. Bachri and H.Aissaoui, Nucl. Phys.B738(2006)7692 hep-ph/0509091.

[26] D. N. Spergel et al., Astophys J. Suppl. 148 (2003)175, astro-ph/0302209.

[27] S. Davidson and A. Ibarra, Phys. Lett.B535(2002)25.

[28] Amal Kr. Sarma, To appear in Eur. Phys. Journal C(2006). 